\title{
HISTOPATOLOGÍA DIFERENCIAL DE TRES ENFERMEDADES BACTERIANAS QUE AFECTAN EL HEPATOPÁNCREAS DE CAMARONES PENEIDOS ${ }^{1}$
}

\author{
Alexander Varela-Mejías ${ }^{2}$,Nelson Peña-Navarro ${ }^{3}$
}

\begin{abstract}
RESUMEN
Histopatología diferencial de tres enfermedades bacterianas que afectan el hepatopáncreas de camarones peneidos. El objetivo del presente trabajo fue caracterizar las lesiones provocadas por las enfermedades necrosis aguda del hepatopáncreas, hepatopancreatitis necrotizante y necrosis séptica del hepatopáncreas, en los camarones de cultivo. Se analizó la información recopilada de fuentes bibliográficas asociadas a observaciones realizadas en muestras de tejidos de camarón infectados. Se utilizó la histopatología como técnica para procesar los tejidos afectados, analizando el tropismo de las lesiones, las particularidades y las características diferenciales entre estas enfermedades. Entre las principales diferencias citadas, las lesiones en la necrosis aguda del hepatopáncreas avanzan desde la región proximal de los túbulos del hepatopáncreas hacia las regiones distales causando desprendimientos celulares masivos y agudos. En la hepatopancreatitis necrotizante se presentan exfoliaciones tubulares de células necróticas con citoplasmas basofílicos y de apariencia granular que dan lugar a la formación de granulomas con atrofia en la fase terminal; y finalmente en la necrosis séptica del hepatopáncreas presenta un avance de lesiones de forma aleatoria con desprendimientos de células epiteliales picnóticas, formación de nódulos hemocíticos y respuesta inflamatoria inmediata. Discernir entre estas patologías brinda la posibilidad de que mediante análisis histopatológicos, se pueda realizar un diagnóstico diferencial entre ellas.
\end{abstract}

Palabras clave: camarón de cultivo, infecciones bacterianas, diagnóstico diferencial.

\begin{abstract}
Differential histopathology of three bacterial diseases that affect the hepatopancreas of penaeid shrimp. The objective of this work was to characterize the injuries caused by the acute hepatopancreatic necrosis; the necrotizing hepatopancreatitis, and septic hepatopancreatic necrosis in shrimp farming. The information collected from many literature sources associated with the observations made in tissue samples from infected shrimp was analyzed. Histopathology was used to process the affected tissues, analyzing tropism of injuries, the special and differential characteristics between these diseases. Among the main differences mentioned: the acute hepatopancreatic necrosis injuries move forward from the proximal areas of the tubules of the hepatopancreas to the distal regions and acute cell causing massive and intensive sloughing. In peels necrotising hepatopancreatitis tubular necrotic cells present with basophilic cytoplasm and granular appearance leading to granulom formation atrophy in the terminal phase; and finally the septic hepatopancreatic necrosis injury presents a randomly advance of injuries with pyknotic epithelial cell detachment, forming hemocytic and immediate inflammatory response. Distinguish between these diseases provides the possibility that, by histopathological analysis can make a differential diagnosis between them.
\end{abstract}

Keywords: shrimp farming, bacterial infections, differential diagnosis.

\footnotetext{
1 Recibido: 3 febrero, 2015. Aceptado: 21 julio, 2015. Este trabajo forma parte del proyecto de investigación "Prevalencia de enfermedades en Litopenaeus vannamei cultivado en Costa Rica" de la Universidad Técnica Nacional, Puntarenas, Costa Rica.

2 Laboratorio de Patologías y Parasitología de Crustáceos. Nicoya, Guanacaste, Costa Rica. alexander.varela@gmail.com

3 Universidad Técnica Nacional de Costa Rica. Apartado postal: 148-5400 Puntarenas.npena@utn.ac.cr
} 


\section{INTRODUCCIÓN}

El impacto de las enfermedades infecciosas en las producciones acuícolas, representa grandes riesgos y genera pérdidas económicas significativas a nivel global (Bradley-Dunlop et al., 2004; Vincent et al., 2004; Morales-Covarrubias et al., 2006; Nunan et al., 2008; Morales-Covarrubias, 2010; Nunan et al., 2013; Peña et al., 2013). Este factor se evidencia en brotes como los causados por el virus del síndrome de la mancha blanca (Lightner, 1996; Varela y Peña, 2014a) o la incidencia de la hepatopancreatitis necrotizante (Vincent y Lotz, 2005; Vincent y Lotz, 2007; MoralesCovarrubias, 2008; Varela y Peña, 2015), y más reciente, con los brotes de la necrosis aguda del hepatopáncreas (Tran et al, 2013; Pantoja y Lightner, 2014; Han et al. 2015a).

Las enfermedades bacteriales han aumentado su incidencia e impacto en forma global (AguirreGuzmán et al., 2005; Varela, 2007; Peña et al., 2013) con el agravante de que algunas de ellas comparten los tejidos blancos.

Durante los últimos años se ha presentado una patología emergente denominada enfermedad de la necrosis hepatopancreática aguda o AHPND (por sus siglas en inglés), la cual fue reportada por primera vez en el año 2009 en camarones de cultivo en China, afectando a las especies Penaeus monodon y Litopenaeus vannamei. Posteriormente, esta enfermedad se propagó a países cercanos, impactando a Vietnam, Malasia y Tailandia, y recientemente fue detectada en México (Tran et al., 2013).

La AHPND es causada por una cepa altamente virulenta de Vibrio parahaemolyticus (Tran et al., 2013; Joshi et al., 2014; Pantoja y Lightner, 2014; Han et al., 2015a). Esta cepa bacterial es portadora de un plásmido de aproximadamente $69 \mathrm{~kb}$, responsable de la síntesis de toxinas con actividad proteolítica, las cuales dañan en forma irreversible al hepatopáncreas, generando desprendimientos celulares masivos. Las bacterias portadoras poseen desde unas pocas copias del plásmido hasta más de una centena, lo cual ha sido determinado mediante PCR cuantitativo (Han et al., 2015b).

Previo a AHPND, al menos otras dos enfermedades bacteriales han demostrado tener la capacidad de lesionar el hepatopáncreas, estas son: la hepatopancreatitis necrotizante o NHP (por sus siglas en inglés) (Lightner, 1996; Vincent y Lotz, 2005; Nunan et al., 2008; Morales-Covarrubias, 2008;
OIE, 2012) y la necrosis séptica del hepatopáncreas o SHPND (por sus siglas en inglés) (Lightner, 1996; Cuéllar-Anjel, 2008; Morales-Covarrubias, 2013).

La NHP fue inicialmente denominada hepatopáncreas granulomatoso. Esta patología ha sido la causa de eventos infecciosos de gran impacto en América. Se detectó inicialmente en Texas, USA, en 1985; posteriormente, se propagó a Centro y Sur América (Lightner, 1996; Bradley-Dunlop et al., 2004; Crabtree et al., 2006; Vincent y Lotz, 2007; MoralesCovarrubias, 2008; Morales-Covarrubias, 2010; Varela y Peña, 2015).

La infección del NHP es causada por bacterias intracelulares obligadas, recientemente clasificadas como rickettsias, bajo el nombre propuesto de Hepatobacter penaei (Nunan et al., 2013). Se trata de bacterias Gram negativas, móviles mediante ocho flagelos localizados en su ápex basal. Presentan dos variantes morfológicas, la forma helicoidal, con dimensiones de $0,25 \mu \mathrm{m}$ por $3,5 \mu \mathrm{m}$, y la forma bacilar predominante con $0,25 \mu \mathrm{m}$ por $0,9 \mu \mathrm{m}$ (Lightner, 1996; Nunan et al., 2013); estas bacterias infectan, se multiplican y acumulan en el citoplasma de las células epiteliales de los túbulos del hepatopáncreas (Lightner, 1996; Vincent et al., 2004; Crabtree et al., 2006; Morales-Covarrubias et al., 2006; Vincent y Lotz, 2007; Morales-Covarrubias, 2008), alcanzando concentraciones sumamente altas en los tejidos afectados, en infecciones letales se han determinado concentraciones del orden de $10^{6}$ a $10^{7}$ copias $/ \mathrm{mg}$ de hepatopáncreas (Vincent y Lotz, 2005).

La SHPN es causada por bacterias extracelulares, generalmente pertenecientes al género Vibrio, Gram negativas, mótiles, individuales, las cuales son comunes en ambientes marinos y estuarinos, y pueden provocar mortalidades masivas en los cultivos afectados, generando daños severos en diferentes tejidos, incluyendo los túbulos hepatopancreáticos, asociados a fuertes respuestas de tipo inflamatorio (Lightner, 1996; Cuéllar-Anjel, 2008; MoralesCovarrubias, 2010).

En algunos países como Costa Rica, el monitoreo sanitario se realiza principalmente basado en los signos clínicos de los animales, mediante la realización de análisis en fresco, lo cual representa una gran limitante por su baja sensibilidad y especificidad.

La signología clínica de estas tres patologías comparten similitudes; como lo son una marcada palidez del hepatopáncreas, el cual presenta atrofia, 
el intestino de los animales infectados se muestra vacío o con contenido entrecortado, anorexia, letargia, y ocasionalmente infestaciones por parasitologías secundarias, siendo común la presencia de aves cazando en los estanques afectados (Lightner, 1996; Bradley-Dunlop et al., 2004; Vincent et al., 2004; Morales-Covarrubias, 2008; Cuéllar-Anjel et al., 2012; Varela y Peña, 2015).

El objetivo del presente trabajo fue caracterizar las lesiones provocadas por las enfermedades necrosis aguda del hepatopáncreas, hepatopancreatitis necrotizante y necrosis séptica del hepatopáncreas, en los camarones de cultivo.

\section{MATERIALES Y MÉTODOS}

Camarones de estudio: los juveniles de $L$. vannamei utilizados para el estudio de NHP y SHPND se obtuvieron en la zona de Colorado de Abangares, Guanacaste, Costa Rica y provenían de monitoreos sanitarios aplicados en la región. Las fotografías de AHPND fueron tomadas a partir de la colección de material histológico del laboratorio de la Universidad de Arizona (UAZ).

Histología: para la fijación y preparación de los animales e histología, se siguió la metodología descrita por Bell y Lightner (1988) y Cuéllar-Anjel (2008). Se realizaron cortes sagitales de los hepatopáncreas de los camarones. Por último, se realizó la tinción de todos los tejidos con eosina y hematoxilina (Lightner, 1996; Morales-Covarrubias, 2010). Estos se analizaron observándolos en un microscopio óptico a aumentos de 40, 100 y 400x.

El procesamiento de los tejidos utilizados para el análisis histopatológico se realizó en Costa Rica, en la sección de patología del Laboratorio Nacional de Servicios Veterinarios (LANASEVE), Servicio Nacional de Salud Animal (SENASA) del Ministerio de Agricultura y Ganadería (MAG).

\section{RESULTADOS Y DISCUSIÓN}

\section{Enfermedad de la necrosis hepatopancreática aguda (AHPND)}

Las infecciones por AHPND presentaron tres fases secuenciales definidas histopatológicamente
(Morales-Covarrubias y Gómez-Gil, 2014; Pantoja, 2014, Varela y Peña, 2014b), en la fase aguda o inicial se observó la pérdida de la funcionalidad de las células epiteliales B, F y R de los túbulos del hepatopáncreas, lo que se mostró como ausencia de diferenciación celular. Las células E cesaron su división. Hubo un desprendimiento masivo de estas células, siguiendo un patrón definido, este inició en las zonas proximales de los túbulos avanzando hacia las zonas distales, finalmente se dio el desprendimiento epitelial en ausencia de bacterias de cualquier tipo (Pantoja y Lightner, 2014; Han et al. 2015b) (Figura 1A).

Muchas de las células desprendidas hacia el lumen tubular presentaron núcleos sin alteraciones evidentes, algunas células E mostraron cariomegalia. En esta fase, las bacterias de la cepa patogénica de $V$. parahaemolyticus se encontraron en el estómago, lugar desde el cual liberan las toxinas que lesionan los túbulos y no se observaron bacterias en los cortes del hepatopáncreas (Tran et al., 2013).

Durante la fase de transición se observó, por primera vez, presencia de bacterias en cantidades moderadas, generalmente se iniciaron infecciones secundarias por bacterias oportunistas y se dio lugar al proceso de melanización en los túbulos fuertemente necrosados (Pantoja y Lightner, 2014) (Figura 1B).

Finalmente, durante la fase terminal (Figura 2) de la infección, se generalizó la destrucción del hepatopáncreas por la acción bacterial, con una severa infiltración hemocítica intra y extratubular acompañada de melanosis y necrosis, y se presentaron infecciones bacteriales secundarias masivas y multifocales (Cuéllar-Anjel et al., 2012; Joshi et al., 2014; Tran et al., 2013; Pantoja, 2014; Pantoja y Lightner, 2014; Varela y Peña, 2014b).

\section{Hepatopancreatitis necrotizante (NHP)}

Los tejidos procesados afectados por infecciones por NHP, demostraron inicialmente una disminución de las reservas lipídicas, exfoliaciones tubulares de células cuyos núcleos habían sido desplazados hacia la periferia celular (Figura 3A). En algunas células se observó material granular en los citoplasmas, constituido por masas de bacterias de células basofílicas rodeadas de hemocitos y se presentó la formación de granulomas (Figura 3B) y necrosis tubular (Lightner, 1996; Morales-Covarrubias, 2008; Morales-Covarrubias, 2010). 

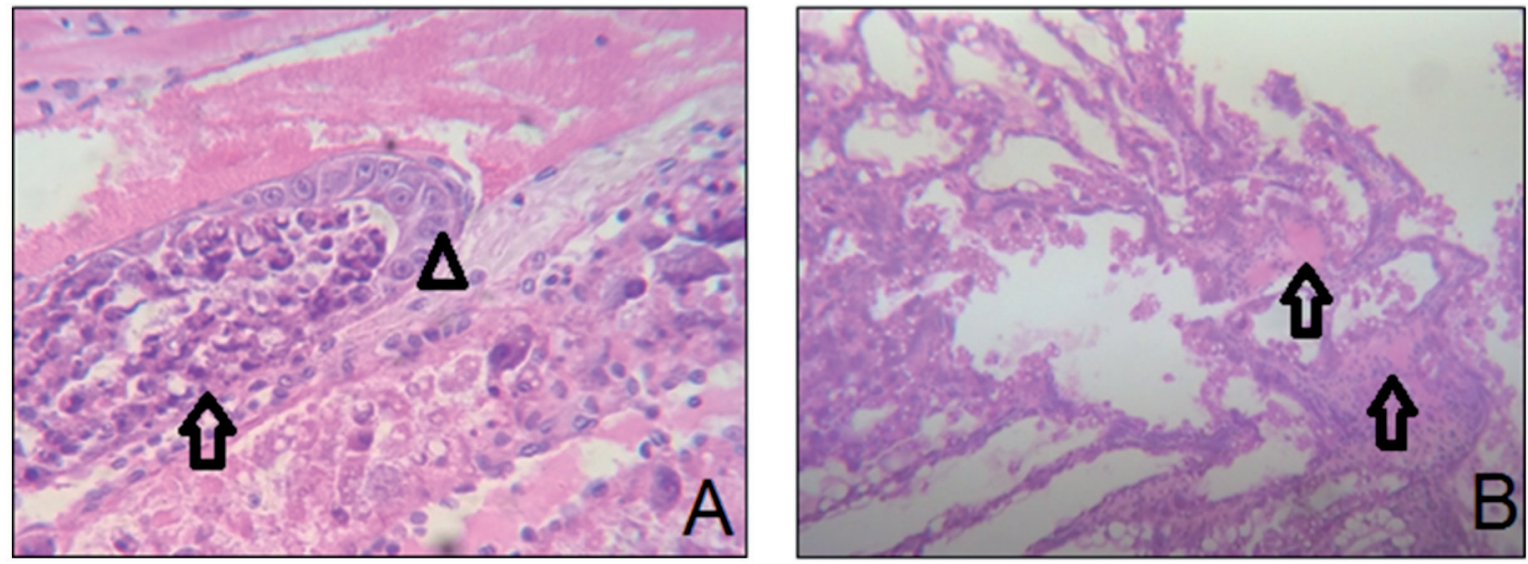

Figura 1. Hepatopáncreas con necrosis hepatopancreática aguda (AHPND). A. Fase inicial: corte de hepatopáncreas, se observa un túbulo con desprendimiento severo de células epiteliales en la región media (flecha). La región apical del túbulo no presenta desprendimientos, pero si una marcada disminución de actividad mitótica (cabeza de flecha) (400X). B. Fase de transición: es posible en algunos casos la detección de masas bacteriales, adicionalmente se observan lesiones tubulares en las regiones proximales y mediales. (Flechas) Continúa el desprendimiento celular y no se observan reservas lipídicas (100X).

Figure 1. Hepatopancreas with AHPND. A. Initial phase: Histological section of hepatopancreas, a tubule with severe epithelial cell sloughing in the middle region (arrow) is observed. The apical region of tubule do not show apparent injuries, but a decreased mitotic activity is present (Arrow head) (400X). B. Transition phase: detection of bacterial masses is possible, also tubular lesions in proximal and medial regions are observed (arrows). Cell sloughing continues and no lipid reserves are observed (100X).

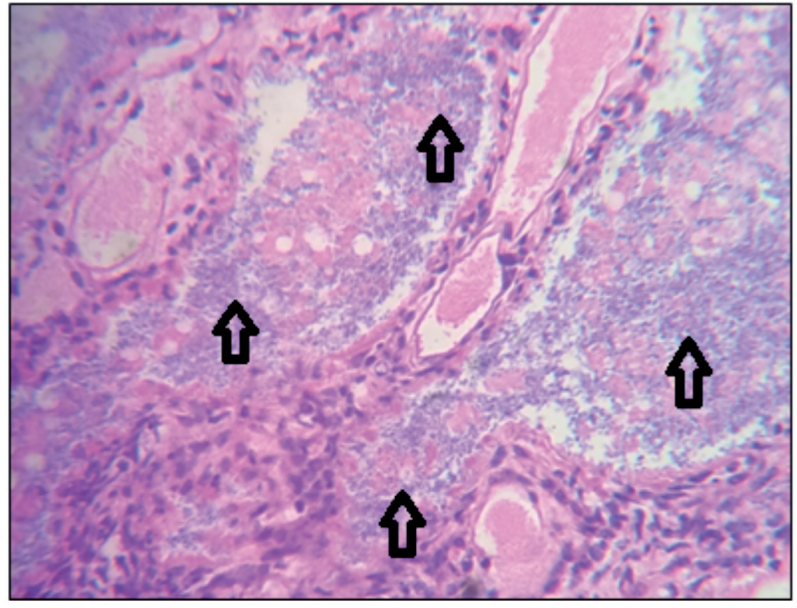

Figura 2. Hepatopáncreas con necrosis hepatopancreática aguda (AHPND) en fase terminal. Corte de hepatopáncreas, en él la invasión bacterial se generaliza (flechas), no se observan túbulos normales y hay una pérdida total de la estructura del órgano. 400X.

Figure 2. Hepatopancreas with acute hepatopancreatic necrosis AHPND in terminal phase. Histological section of hepatopancreas, it generalizes the bacterial invasion (arrows), abnormal tubules are not observed, and there is a total loss of organ structure. $400 \mathrm{X}$.
La distribución de las lesiones no siguió un patrón definido, túbulos afectados podrían estar rodeados de tejido sano. Durante la fase crónica o terminal de la infección, la respuesta inflamatoria del hospedador disminuyó hasta desaparecer, los túbulos se atrofiaron y se presentaron regiones edematizadas (Morales-Covarrubias, 2008).

\section{Necrosis séptica del hepatopáncreas (SHPN)}

SHPN presentó comúnmente desprendimientos de células epiteliales, las cuales estaban hipertrofiadas con los núcleos picnóticos y citoplasmas hipereosinofílicos. Se formaron nódulos hemocíticos, los cuales podrían estar melanizados (Figura 4A), hubo necrosis tubular y una fuerte infiltración hemocítica (Lightner, 1996; Cuéllar-Anjel, 2008, Morales-Covarrubias, 2010). Se detectaron bacterias extracelulares individuales o en cúmulos, que se presentaron como masas basofílicas en los espacios intertubulares (Varela y Peña, 2014b). Por otro lado, se observaron cápsulas rodeando túbulos necrosados (Figura 4B). El progreso de las lesiones no presentó un patrón definido. La distribución de las lesiones y nódulos hemocíticos fue "aleatoria" en el órgano (Pantoja, 2014). 

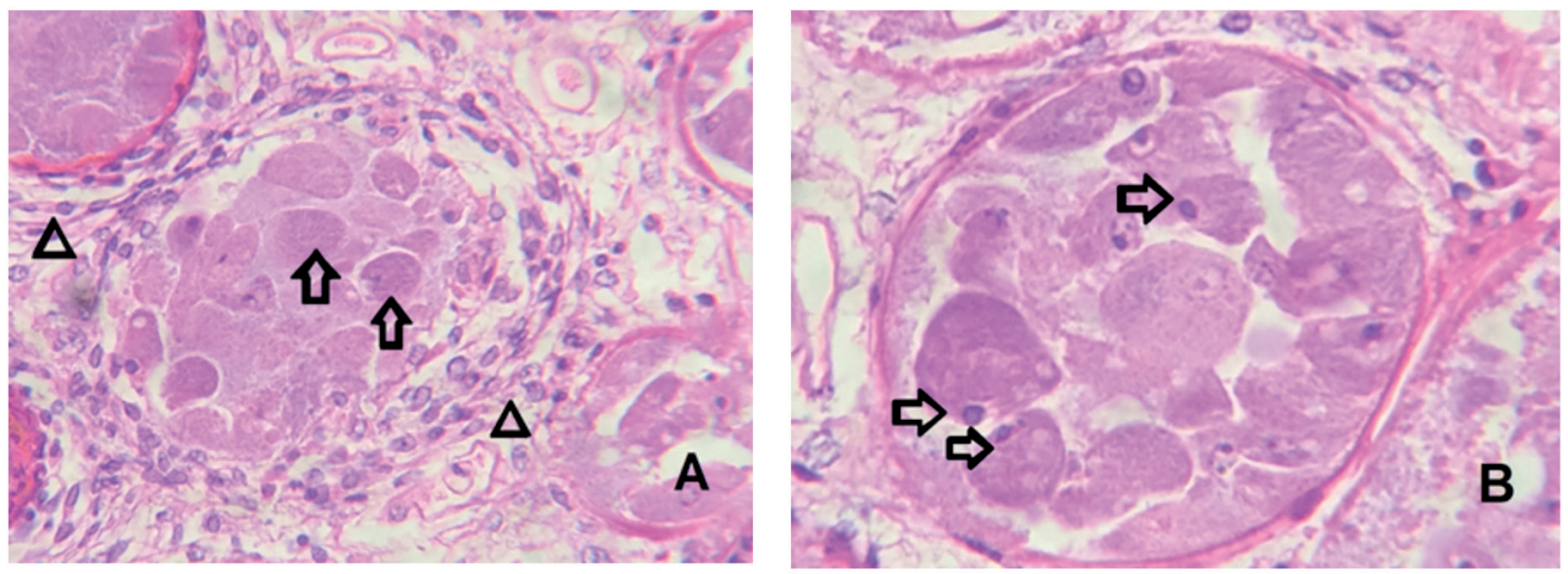

Figura 3. Hepatopáncreas con hepatopancreatitis necrotizante. A. Exfoliaciones de células epiteliales de los túbulos, estas lucen basofílicas debido a la replicación bacterial en los citoplasmas dando apariencia granular (flechas), las lesiones están rodeadas de hemocitos (cabeza de flecha), la formación de granulomas y necrosis tubular es común. 100X. B. Se observa una leve disminución de vacuolas, se observan múltiples células desprendidas en el lumen tubular (flecha), presentando núcleos picnóticos y en muchos casos excéntricos. 100X.

Figure 3. Hepatopancreas with necrotizing hepatopancreatitis (NHP). A. Exfoliations of tubule epithelial cells, these look basophilic due to bacterial replication in the cytoplasm giving a granular appearance (arrows), lesions are surrounded by hemocytes (arrow head), granuloma formation and tubular necrosis is common. 100X. B. A slight decrease in lipid vacuoles were observed, a detached cell moves through the tubular lumen (arrows), with pyknotic and many cases with eccentric nucleus. 100X.
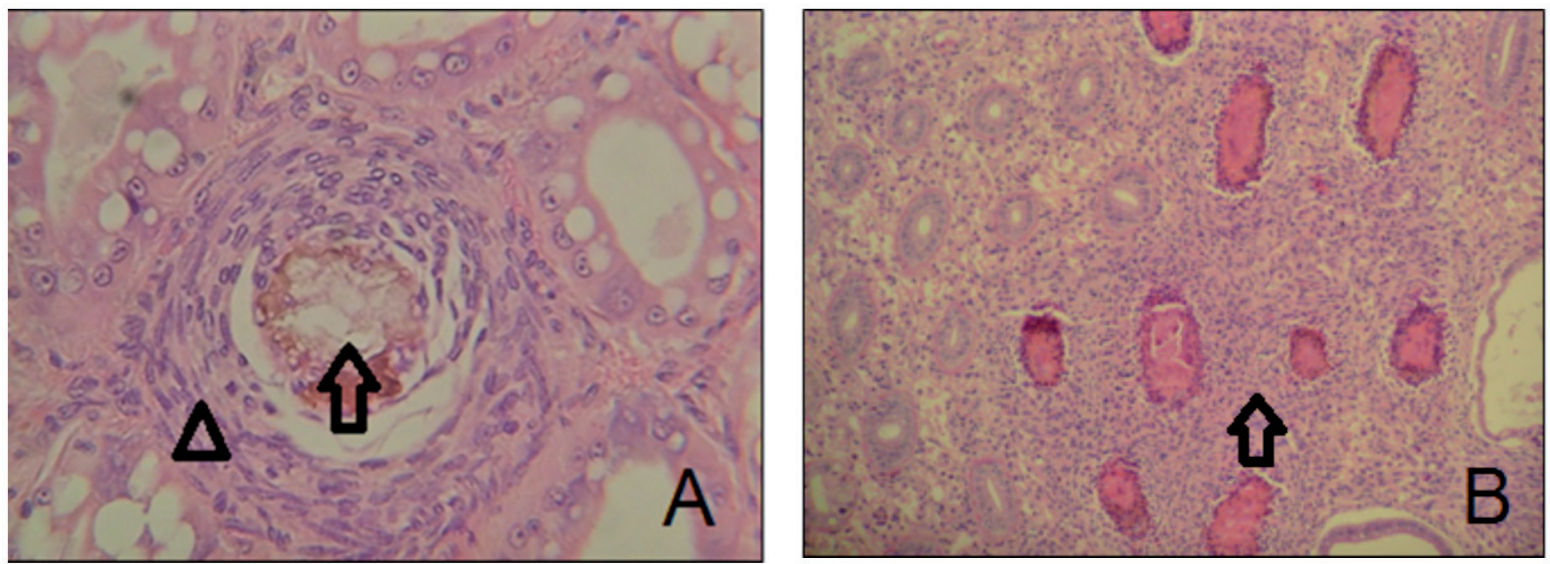

Figura 4. Hepatopáncreas con necrosis séptica del hepatopancreas. A. Nódulo hemocítico melanizado en espacio intertubular (flecha), se observa el patrón concéntrico de los hemocitos (cabeza de flecha), los túbulos adyacentes no presentan lesiones aparentes 100X. B. Cápsulas rodeando túbulos necróticos melanizados, infiltración hemocítica masiva (flecha). 40X.

Figure 4. Hepatopancreas with septic hepatopancreatic necrosis (SHPND). A. Melanized hemocytic nodule in intertubular space (arrow), concentric pattern of the hemocytes (arrow head), is observed adjacent tubules have no apparent injuries 100X. B. Capsules encircling necrotic tubules and haemocytic massive infiltration with inflammatory response (arrow). 40X. 
Con el fin de enfatizar las principales características de estas patologías, se presenta un cuadro de referencia con el fin de destacar algunas de las principales diferencias entre estas tres enfermedades (Cuadro 1).

Los resultados obtenidos mediante técnicas de histopatología son altamente sensibles y pueden ser muy específicos, para ello, el analista debe estar familiarizado con las lesiones de cada una de las patologías de modo que sea factible detectar infecciones individuales o combinadas.

El AHPND se presenta como una patología que genera lesiones severas en periodos de tiempos cortos, siendo por tanto una patología aguda, capaz de generar pérdidas de población desde los periodos iniciales de los cultivos. La ausencia inicial de bacterias en el hepatopáncreas, imposibilita su recuperación y cultivo in vitro partiendo de muestras de este órgano. El NHP se caracteriza por ser una enfermedad de tipo crónico, sus efectos en la población se presentan a lo largo del ciclo productivo, las lesiones son de tipo focal o multifocal. Por tratarse de rickettsias, no se dispone en la actualidad de medios de cultivo in vitro.
Las infecciones por SHPND desarrollan lesiones asociadas directamente a la presencia bacterial, de modo que es factible observar bacterias desde estadíos iniciales de la infección. También es posible realizar cultivos de muestras de hepatopáncreas desde el inicio de la infección en medios de cultivo in vitro.

Finalmente, ante sospechas sobre la causa de la infección o la posibilidad de infecciones múltiples, es recomendable realizar análisis complementarios de alta sensibilidad y especificidad, como la reacción en cadena de polimerasa (PCR).

\section{AGRADECIMIENTO}

Al laboratorio de patología acuícola de la Universidad de Arizona, por facilitar el material histológico a partir del cual, se capturaron las imágenes de AHPND. Al Laboratorio Nacional de Servicios Veterinarios (LANASEVE) por facilitar el equipo utilizado para realizar los análisis histológicos.

Cuadro 1. Resumen de las diferencias entre tres enfermedades bacteriales de mayor incidencia en los cultivos comerciales de Litopenaeus vannamei. Modificado de Pantoja y Lightner (2014).

Table 1. Summary of differences between the three bacterial diseases of greatest incidence in Litopenaeus vannamei commercial crops. Modified from Pantoja \& Lightner (2014).

Enfermedad

Necrosis aguda del hepatopáncreas (AHPND) Muchas de las células desprendidas presentan núcleos con apariencia cuasi-normal. Ausencia de cualquier tipo de bacterias en estadíos tempranos de la infección, en fases avanzadas hay comúnmente infecciones secundarias y necrosis masiva.

Hepatopancreatitis necrotizante (NHP)

Descripción de las principales lesiones causadas por tres enfermedades bacteriales que afectan el hepatopáncreas de camarones peneidos

Inicialmente se observa una disminución de las reservas lipídicas, hay exfoliaciones tubulares de células con citoplasmas basofílicos y de apariencia granular, rodeadas de hemocitos. Presencia focal o multifocal de granulomas. En infecciones avanzadas se observa atrofia y disminución de la altura epitelial. En muchos casos acompañado de la formación de edemas y ausencia de respuesta inflamatoria.

Necrosis séptica del hepatopáncreas (SHPN) Avance de lesiones de forma aleatoria. Desprendimiento de células hipertrofiadas, con núcleos picnóticos, necrosis tubular con y fuerte infiltración hemocítica. Presencia de nódulos hemocíticos, los cuales pueden estar melanizados. Presencia bacterial desde el inicio de la infección, las cuales se observan libres o en cúmulos. 


\section{LITERATURA CITADA}

Aguirre-Guzmán, G., F. Ascencio, and D. Saulnier. 2005. Pathogenicity of Vibrio penaeicida for white shrimp Litopenaeus vannamei: a cysteine protease-like exotoxin as a virulence factor. Dis. Aquat. Org. 67: 201-207.

Bell, T. A., and D.V. Lightner, 1988. A handbook of normal penaeid shrimp histology. World Aquaculture Society, Baton Rouge, LA, USA.

Bradley-Dunlop, B.J., C. Pantoja, and D.V. Lightner. 2004. Development of monoclonal antibodies for detection of necrotizing hepatopancreatitis in penaeid shrimp. Dis. Aquat. Org. 60:233-240.

Crabtree, B.G., M.M. Erdman, D.L. Harris, and I. Turney. 2006. Preservation of necrotizing hepatopancreatitis bacterium (NHPB) by freezing tissue collected from experimentally infected Litopenaeus vannamei. Dis. Aquat. Org. 70:175-179.

Cuéllar-Anjel, J. 2008. Métodos de diagnósticos de enfermedades en camarones marinos de cultivo. En: V. Morales, y J. Cuéllar- Anjel, editores, Guía técnica: patología e inmunología de camarones penaeidos. Programa CYTED Red II-D Vannamei, PAN. p. 1-54.

Cuéllar-Angel, J., D.V. Lightner., y C. Pantoja. 2012. Síndrome de mortalidad temprana o síndrome de necrosis hepatopancreática aguda. Panorama Acuícola Magazine 17:42-43.

Han, J.E., L. Mohney, K. Tang, C. Pantoja, and D.V. Lightner. 2015a. Plasmid mediated tetracycline resistance of Vibrio parahaemolyticus associated with acute hepatopancreatic necrosis disease (AHPND) in shrimps. Aquaculture Reports 2:17-21.

Han, J.E., K. Tang, L. Tran, and D. Lightner. 2015 b. Photorhabdus insect-related (Pir) toxin-like genes in a plasmid of Vibrio parahaemolyticus, the causative agent of acute hepatopancreatic necrosis disease (AHPND) of shrimp. Dis. Aquat. Org. 113:33-40.

Joshi, J., J. Srisala, V.H. Truong, I.T. Chen, B. Nuangsaeng, O. Suthienkul, C.F. Lo, T.W. Flegel, K. Sritunyalucksana, and S. Thitamadee. 2014. Variation in Vibrio parahaemolyticus isolates from a single Thai shrimp farm experiencing an outbreak of acute hepatopancreatic necrosis disease (AHPND). Aquaculture (428-429):297-302.

Lightner, D.V. 1996. A handbook of shrimp pathology and diagnostic procedures for diseases of cultured penaeid shrimp. World Aquaculture Society, Baton Rouge, LA, USA.
Morales-Covarrubias, M.S. 2008. Enfermedades bacteriales. En: V. Morales, y J. Cuéllar- Anjel, editores, Guía técnica: patología e inmunología de camarones peneidos. Programa CYTED Red II-D Vannamei, PAN. p. 117-134.

Morales-Covarrubias, M.S. 2010. Enfermedades del camarón: detección mediante análisis en fresco e histopatología. Editorial Trillas. México, D.F., MEX.

Morales-Covarrubias, M.S. 2013. Camarón análisis en fresco, herramienta de diagnóstico. 1era. edición. CIAD-OIRSA, MEX.

Morales-Covarrubias, M.S., A.G. Osuna, A. García, D.V. Lightner, and J.C. Mota. 2006. Prevalence of necrotizing in female broodstock of white shrimp Penaeus vannamei with unilatral eyestalk ablation and hormone injection. J. Aquatic Anim. Health 18:19-25.

Morales-Covarrubias, M.S, y B. Gómez-Gil. 2014. Enfermedades bacterianas de camarones. En: V. Morales, y J. Cuéllar-Anjel, editores, Guía técnica: patología e inmunología de camarones penaeidos, $2 \mathrm{da}$ ed. OIRSA, Rep. de Panamá, PAN. p. 167-171.

Nunan, L., C. Pantoja, S. Gómez-Jiménez, and D.V. Lightner. 2013. "Candidatus Hepatobacter penaei" an shrimp Penaeus vannamei in the Hepatopancreas of the marine intracellular pathogenic enteric bacterium (Crustacea: Decapoda). Appl. Environ. Microbiol. 79(4):1407.

Nunan, L.M., C. Pantoja, and D.V. Lightner. 2008. Improvement of a PCR method for the detection of necrotizing hepatopancreatitis in shrimp. Dis. Aquat. Org. 80:69-73.

OIE (Organización Mundial de Sanidad Animal). 2012. Manual de diagnóstico en animales acuáticos. 6 ed., OIE, Paris, FRA.

Pantoja, C. 2014. Otras enfermedades bacterianas que afectan el hepatopáncreas del camarón peneido (EMSdiagnóstico diferencial). Presentación durante el $3^{\text {er }}$ Curso teórico práctico de actualización en patología e inmunología del camarón blanco Litopenaeus vannamei. OIRSA, PAN.

Pantoja, C., y D.V. Lightner. 2014. EMS/AHPND: Descripción de la enfermedad en Asia y América. En: Morales, V. y J. Cuéllar-Anjel, editores, Guía técnica: patología e inmunología de camarones penaeidos. OIRSA, Rep. de Panamá, PAN. p.172-177.

Peña, N., R. Vargas, y A. Varela. 2013. Productos naturales como estimuladores del sistema inmunológico de Litopenaeus vannamei, infectado con Vibrio parahaemolyticus. Agron. Mesoam. 24:133-147.

Tran, L., L. Nunan, R. Redman, L. Mohney, C. Pantoja, K. Fitzsimmons, and D.V. Lightner. 2013. Determination of the infectious nature of the agent of acute 
hepatopancreatic necrosis syndrome affecting penaeid shrimp. Dis. Aquat. Org. 105:45-55.

Varela, A. 2007. Manual para la interpretación de resultados de laboratorio. Alicorp, Publicis- Asociados, PER.

Varela, A., y N. Peña. 2014a. Fluctuaciones térmicas y su relación con el virus del síndrome de las manchas en Litopenaeus vannamei cultivado en el Golfo de Nicoya“ Rev. UTN Informa 68:80-83.

Varela, A., y N. Peña. 2014b. Síndrome de la mortalidad temprana (EMS/AHPNS) en camarones cultivados: Una revisión. Repertorio Científico 17:25-30.

Varela, A., y N. Peña. 2015. Hepatopancreatitis Necrotizante asociada al Fenómeno del Niño, en cultivos de camarones del Golfo de Nicoya. Repertorio Científico 18:29-34.
Vincent, A., Breland, V.M, and J. Lotz. 2004. Experimental infection of Pacific white shrimp Litopenaeus vannamei with Necrotizing Hepatopancreatitis (NHP) bacterium by per os exposure. Dis. Aquat. Org. 61:227-233.

Vincent, A., and J. Lotz. 2005. Time course of necrotizing hepatopancreatitis (NHP) in experimentally infected Litopenaeus vannamei and quantification of NHPbacterium using real-time PCR. Dis. Aquat. Org. 67:163-169.

Vincent, A., and J. Lotz. 2007. Effect of salinity on transmission of necrotizing hepatopancreatitis bacterium (NHPB) to Kona stock Litopenaeus vannamei. Dis. Aquat. Org. 75:265-268. 